ARQGA / 935

\title{
AVALIAÇÃO DO TESTE RÁPIDO DA UREASE CONSERVADO EM GELADEIRA
}

\author{
Laura Cotta ORNELLAS*, Marcelo de Souza CURY**, \\ Vinícius Machado de LIMA** e Angelo Paulo FERRARI Jr.***
}

RESUMO - O teste rápido da urease, método acurado, barato, de resultado prontamente acessível, vem sendo largamente utilizado na pesquisa do Helicobacter pylori. Com qualidade equivalente à dos testes comerciais, o teste rápido da urease não tamponado tem sido preparado para uso diário. Objetivo - Avaliação deste método estocado em geladeira ( $\left.4^{\circ} \mathrm{C}\right)$. Material e método - Foram obtidas biopsias endoscópicas da mucosa antral de 104 pacientes consecutivos, nas quais a pesquisa do Helicobacter pylori foi realizada pelo teste rápido da urease, exame anatomopatológico e teste rápido da urease estocado mantido em geladeira por periodo de 1 a 8 dias e retirado no dia do exame. Resultados - Consideramos presente a infecção quando o teste rápido da urease e anatomopatológico foram concomitantemente positivos, o que ocorreu em 45 pacientes (42\%). O teste rápido da urease estocado mostrou especificidade comparável ao teste rápido da urease (93\%), com sensibilidade de 88\%. Conclusão - Podemos concluir que o teste rápido da urease estocado mantém acurácia adequada, permitindo seu uso rotineiro. Assim, torna-se ainda mais fácil a utilização de testes de urease não tamponados, já que a acurácia diagnóstica fica mantida mesmo com estocagem por até 8 dias.

DESCRITORES - Endoscopia gastrointestinal. Urease. Helicobacter pylori.

\section{INTRODUÇÃO}

O Helicobacter pylori, um bacilo gram-negativo patogênico produtor de urease, isolado inicialmente em $1982^{(10)}$, tem se mostrado intimamente relacionado à úlcera péptica duodenal e gástrica, duodenite erosiva, gastrite crônica e também ao câncer gástrico. $\mathrm{O}$ teste rápido da urease (TRU), um dos métodos para sua detecção na biopsia endoscópica, é de fácil execução, baixo custo e resultado prontamente acessível. Apesar das várias preparações disponíveis no mercado, os testes de urease não tamponados têm mostrado acurácia comparável e custo cumulativo menor ${ }^{(1)}$, sendo largamente utilizados na prática clínica.

Devido à possibilidade de contaminação por bactérias produtoras de urease como Proteus sp e Pseudomonas $s p$, levando a alterações na cor do teste durante a estocagem, preconiza-se que a preparação contendo uréia e o marcador sensível de $\mathrm{pH}$ seja feita diariamente. Trabalhos anteriores ${ }^{(3,6)}$ têm sugerido que o TRU possa ser estocado a baixas temperaturas por tempo limitado, preservando sua

Setor de Endoscopia Digestiva, Disciplina de Gastroenterologia, Departamento de Medicina, Universidade Federal de São Paulo - UNIFESP.

* Pós-graduanda da Disciplina de Gastroenterologia da UNIFESP.

** Residente da Disciplina de Gastroenterologia da UNIFESP.

*** Professor Adjunto da Disciplina de Gastroenterologia da UNIFESP.

Endereço para correspondência: Dr. Angelo Paulo Ferrari Jr. - Disciplina de Gastroenterologia - UNIFESP - Rua Pedro de Toledo, 980 , Cj. 66 - 04039-002 - São Paulo, SP. e-mail: Angelo@gastro.epm.br 
sensibilidade. Deste modo, este estudo objetiva a avaliação prospectiva da acurácia do teste rápido da urease estocado (TRUE) a $4^{\circ} \mathrm{C}$.

\section{PACIENTES E MÉTODO}

Entre os pacientes submetidos a endoscopia digestiva alta no setor de Endoscopia da Disciplina de Gastroenterologia da Universidade Federal de São Paulo (UNIFESP-EPM), no período de junho a agosto de 1998, foram incluídos, aleatoriamente, 104 pacientes, excluindo-se apenas aqueles gastrectomizados. Os pacientes submetidos a terapia de erradicação do Helicobacter pylori não foram excluídos. A endoscopia digestiva alta foi realizada sob sedação individualizada (midazolam e/ou meperidina), após jejum de 10 horas.

Em cada paciente foram realizadas seis biopsias do antro gástrico, sendo preparados três frascos com dois fragmentos cada. O frasco I continha o TRU preparado do dia do exame. O frasco II correspondia ao teste rápido previamente preparado e estocado (TRUE) a temperatura de $4{ }^{\circ} \mathrm{C}$ por um período variável ( 1 a 8 dias). Este foi retirado da geladeira uma hora antes do exame a fim de ser utilizado à temperatura ambiente. O frasco III com formol a $10 \%$, foi encaminhado para exame anatomopatológico (AP) realizado pelo Departamento de Anatomia Patológica da UNIFESP sem o conhecimento do resultado dos outros testes. O material foi corado pela coloração de Giemsa modificada. Consideramos o Helicobacter pylori presente quando tanto o TRU, como o AP foram positivos.

$\mathrm{O}$ teste rápido da urease foi preparado com $1 \mathrm{~mL}$ de água destilada, $1 \mathrm{mg}$ de uréia a $10 \%$ e duas gotas de fenol vermelho a $1 \%$ e considerado positivo se a mudança de cor ocorrer em até 5 minutos, segundo descrição prévia ${ }^{(9)}$.

\section{RESULTADOS}

Dentre os 104 pacientes incluídos, 58 eram do sexo masculino e 46 do sexo feminino, com idade variando de 14 a 81 anos (média 44 e mediana 45 anos). Os diagnósticos mais freqüentes são apresentados na Tabela 1. Em alguns pacientes, a endoscopia mostrou mais de uma lesão.

O Helicobacter pylori estava presente em 45 pacientes. O TRU (frasco I) apresentou quatro falso positivos e nenhum falso negativo. O TRUE (frasco II) apresentou quatro falso positivo e cinco falso negativos e ocorreram 11 falso positivos e nenhum falso negativo no AP (frasco III). Os resultados para sensibilidade, especificidade, valor preditivo positivo e negativo e acurácia são apresentados na Tabela 2.

\section{DISCUSSÃO}

Apesar da variedade de técnicas disponíveis para o diagnóstico da infecção pelo Helicobacter pylori, um teste ideal precisa ser simples, seguro, rápido, barato, acurado e facilmente disponível para aplicação
TABELA 1 - Diagnósticos endoscópicos mais freqüentes nos 104 pacientes. Alguns apresentaram concomitância de lesões

\begin{tabular}{lc}
\hline Gastrite & 69 \\
Esofagite & 42 \\
Duodenite & 29 \\
Úlcera duodenal & 26 \\
Úlcera gástrica & 14 \\
Varizes & 13 \\
Normal & 10 \\
Outros & 5 \\
\hline
\end{tabular}

TABELA 2 - Resultados dos 3 testes, considerando como método ouro para o diagnóstico da infecção pelo Helicobacter pylori, a positividade concomitante do AP e TRU. Valores expressos em porcentagem

\begin{tabular}{lccc}
\hline & TRU & TRUE & AP \\
\hline Sensibilidade & 100 & 88 & 100 \\
Especificidade & 93 & 93 & 82 \\
Valor Preditivo Positivo & 91 & 90 & 79 \\
Valor Preditivo Negativo & 100 & 92 & 100 \\
Acurácia & 96 & 91 & 89 \\
\hline
\end{tabular}

$\mathrm{TRU}=$ teste rápido da urease

TRUE $=$ teste rápido da urease estocado

$\mathrm{AP}=$ anatomopatológico

clínica $^{(1)}$. Assim, não há um teste padrão ouro universalmente aceito. Conta-se com métodos invasivos, que requerem esofagogastroduodenoscopia, como a histologia, cultura e TRU. O Helicobacter pylori pode também ser diagnosticado por teste respiratório e sorologia, considerados não-invasivos por não necessitarem de exame endoscópico ${ }^{(8)}$.

Dentre os testes invasivos, o TRU tem muita aceitação devido a sua facilidade de preparo e realização, baixo custo e rapidez, comparados com o exame histológico ${ }^{(4)}$. Na presença da urease produzida pelo Helicobacter pylori, a uréia é convertida em amônia, resultando em mudança do $\mathrm{pH}$ e, conseqüentemente, da cor da solução. Há muitas preparações comerciais disponíveis para o TRU consideradas relativamente mais baratas quando comparadas à histologia e cultura. Apesar disso, o seu custo cumulativo seria substancial se um grande número de testes fosse realizado. Várias preparações não comerciais, produzidas no local do exame, consideravelmente mais baratas que as comerciais, têm sido descritas ${ }^{(1)}$. O teste rápido da urease e o exame anatomopatológico foram estudados anteriormente pelo nosso grupo ${ }^{(5)}$, apresentando sensibilidade e especificidade altas. 
Entretanto, uma variedade de fatores pode influenciar a rapidez e acurácia do TRU como o número e tamanho das biopsias gástricas, temperatura durante leitura ${ }^{(4)}$, tempo e temperatura de estocagem $^{(6)}$.

No presente estudo, o melhor método entre os três estudados foi o TRU preparado no dia do exame, com acurácia de 96\% (Tabela 2). Entretanto, o TRUE a $4{ }^{\circ} \mathrm{C}$ apresentou especificidade igual à do teste preparado no dia, com queda da sensibilidade para $88 \%$, índice ainda bastante satisfatório. Haja vista a economia para o serviço de endoscopia com o preparo do TRU pelos próprios funcionários (médico ou enfermeira), além da praticidade com a conservação em geladeira e redução do tempo gasto diariamente, este método pode ser muito atrativo para uso rotineiro. KATELARIS et al.(3) já sugeriam, em 1992, que o TRU pudesse ser estocado a baixas temperaturas preservando sua qualidade. $\mathrm{NG}$ et al. ${ }^{(6)}$ também mostraram que o TRU mantinha boa acurácia quando estocado a $4{ }^{\circ} \mathrm{C}$ por até 5 dias, com sensibilidade de $92 \%$ e especificidade de $95 \%$, devendo ser conservado a $-20{ }^{\circ} \mathrm{C}$, se necessário maior tempo de estocagem.

A baixa acurácia da pesquisa histológica do Helicobacter pylori, principalmente sua baixa especificidade, pode ser justificada pela utilização apenas do Giemsa modificado como corante e pela participação de um grupo heterogêneo de patologistas neste estudo.

\section{CONCLUSÃO}

O TRU fabricado localmente e estocado em geladeira a $4{ }^{\circ} \mathrm{C}$ permanece com alta sensibilidade e especificidade, permitindo o seu uso com segurança e praticidade nos serviços de endoscopia digestiva.

Ornellas LC, Cury Mde S, Lima VM de, Ferrari Jr AP. Evaluation of rapid urease test stored in refrigerator. Arq Gastroenterol 2000;37(3):155-157.

ABSTRACT - The rapid urease test is an accurate and cheap method, which results are readily available, and broadly used for routine Helicobacter pylori infection diagnosis. Aim - The evaluation of rapid urease test stored in refrigerator at $4{ }^{\circ} \mathrm{C}(\mathrm{SRUT})$ compared to regular rapid urease test. Patients and Method-Endoscopic biopsies were obtained from gastric antrum in 104 consecutive patients. Diagnosis of Helicobacter pylori infection was accomplished by rapid urease test, histology and rapid urease test stored (kept in refrigerator by a period ranging from 1 to 8 days). Results - Infection was considered present if both rapid urease test and histology were positive. Helicobacter pylori was present in 45/104 patients (42\%). Rapid urease test stored had specificity comparable to rapid urease test (93\%), with sensitivity of $88 \%$. Conclusion - We concluded that rapid urease test can be stored in refrigerator for up to 1 week, without loss of clinical applicability, and that can make the test even easier to use for routine Helicobacter pylori tests in a busy endoscopy unit.

HEADINGS - Endoscopy, gastrointestinal. Urease. Helicobacter pylori.

\section{REFERÊNCIAS BIBLIOGRÁFICAS}

1. Chu KM, Poon R, Tuen HH, Law SYK, Branicki FJ, Wong J. A prospective comparison of locally made rapid urease test and histology for the diagnosis of Helicobacter pylori infection. Gastrointest Endosc 1997;46:503.

2. Conti-Nibali S, Sferlazzas C, Fera MT, Saitta G, Tedeschi A, Magazzù G. Helicobacter pylori infection: a simplified diagnostic approach. Am J Gastroenterol 1990;85:1573.

3. Katelaris PH, Lowe DG, Norbu P. Field evaluation of a rapid, simple and inexpensive urease test for detection of Helicobacter pylori. J Gastroenterol Hepatol 1992;7:569.

4. Laine L, Estrada R, Lewin DN, Cohen H. The influence of warning on rapid urease test results: a prospective evaluation. Gastrointest Endosc 1996;44:429.

5. Morais M, Macedo EP, Silva Jr MR, Rohr MRS, Ferraz MLG, Castro RRO, Della Libera E, Siqueira ES, Brant CQ, Ferrari Jr AP. Comparação entre testes invasivos para o diagnóstico da infecção pelo Helicobacter pylori. Arq Gastroenterol $1997 ; 34: 207$.
6. Ng FH, Wong SY, Ng WF. Storage temperature of the unbuffered rapid urease test. Am J Gastroenterol 1997;92:2230.

7. Nichols L, Sughayer M, DeGirolami PC, Balogh K, Pleskow D, Eichelberger K, Santos M. Evaluation of diagnostic methods for Helicobacter pylori gastritis. Am J Clin Pathol 1991;95:769.

8. Onders RP. Detection methods of Helicobacter pylori: accuracy and costs. Am Surg 1997;63:665.

9. Thillainayagam AV, Arvind AS, Cook RS, Harrison JG, Tabaqchali S, Farthing MJG. Diagnostic efficiency of an ultrarapid endoscopy room test for Helicobacter pylori. Gut 1991;32:467.

10. Warren JR, Marshall BJ. Unidentified curved bacilli on gastric epithelium in active chronic gastritis. Lancet 1983;1:1273. 